\title{
A lower bound on the dimension of minimal positive realizations for discrete-time systems
}

\author{
Luca Benvenuti *
}

\begin{abstract}
In some applications, one is interested in having a state-space realization with nonnegative matrices (positive realization) of a given transfer function and it is known that such a realization may have a dimension strictly larger than the order of the transfer function itself. The aim of this letter is to provide a lower bound on the minimum dimension of a positive realization taking into account some spectral properties of nonnegative matrices.
\end{abstract}

Keywords: Positive realization problem; nonnegative matrices; minimal realization.

\section{Introduction}

A positive linear system is a dynamical system in which states and outputs take nonnegative values for any nonnegative input and nonnegative initial state. Hence, positive systems are used to model phenomena in which the variables assume only nonnegative values, due to the nature of the phenomenon itself. They frequently emerge as models of networks of reservoirs or industrial processes involving chemical reactors, heat exchangers and distillation columns, models of age structured population dynamics, models of water or atmospheric pollution propagation, models of stochastic processes, and models of many other processes commonly used in economy and sociology. Several examples with the related references can be found in [8].

For discrete time, linear and time-invariant (LTI) systems, the nonnegativity constraints on state and output sequences are equivalent to nonnegativity constraints on the entries of the matrices $\{A, b, c\}$ defining the system [23]. The impulse response $h(k)$ of a single - input single-output (SISO) positive system is obviously a nonnegative sequence. The Positive Realization Problem (PRP) consists of the converse question: given a discrete time LTI SISO system (or a transfer function $H(z)=\sum_{k=1}^{\infty} h(k) z^{-k}$ ) with a nonnegative

*Dipartimento di Ingegneria informatica, automatica e gestionale "A. Ruberti", Sapienza Università di Roma, via Ariosto 25, 00185 Roma, Italy. 
impulse response $h(k)$, find necessary and sufficient conditions for the existence of a positive system realizing it. Such a positive system is called a positive state space realization of the given impulse response (or transfer function). Necessary and sufficient conditions for the existence of a positive realization have been given in [1] and [12] in 1996. The PRP is of interest, for example, to compartmental analysis [24], digital filter design using optical fibers [7] or charge-coupled devices (CCDs) [3, 15] and identifiability in Hidden Markov Models (HMMs) [2]. As discussed below, the main question in these applications is the characterization of minimality for the positive realizations.

Compartmental systems are effectively used in an impressive number of different applicative fields of mathematical modeling, ranging from tracer kinetics to population dynamics. They are composed of a finite number of subsystems, called compartments, interacting by exchanging material. Since the state variables represent the amount of material contained in each compartment, they are bounded to be nonnegative over time. Then, compartmental systems belong to the class of positive systems. One of the most important problem in the analysis of compartmental systems is the determination of the internal structure of the systems - specifically the number of compartments - from the impulse response obtained through an input-output experiment [24]. In other words, the number of compartments to be extracted from the measured data is not fixed a priori and only the compartmental nature of the model is assumed. An immediate application of this problem in clinical medicine, and specifically in tracer kinetics, is the determination of the number of organs involved in some tracer experiment.

CCDs offer the possibility of achieving digital signal processing on a MOS integrated circuit chip with the advantage of lighter weight, smaller size, lower power consumption and improved reliability with respect to an equivalent standard implementation. They are based on a family of functional solid-state electronic devices that under the application of a sequence of clock pulses move quantities of electrical charge in a controlled manner across a semiconductor substrate. In particular, the devices consist of several locations on the substrate where packets of charge can be stored and maintained isolated from the others, and of a specific sequence of operations - periodically repeating - involving the packets of charge stored in these locations. In this case, the state variables represent the quantities of electric charge in the locations and are bounded to be nonnegative over time. Then a CCD implementation of a given transfer function calls for a positive realization of it. Obviously, when implementing a filter with this technology, one wishes to reduce space occupation and power consumption so that the minimal number of locations is required.

Finally, the PRP is related to the identifiability problem for hidden Markov models (HMMs), that is the problem of realizing an HMM given the collection of probabilities of output strings. This problem is a complication of the positive realization problem since it is a multivariable problem with some additional constraints. Nevertheless, the technique used to construct a solution for the positive realization problem can be built on for solving 
the realization problem for HMMs [2]. Even in this case, an interesting problem is that of determining the minimum number of states needed to realize the collection of probabilities [31].

These considerations alone justify an effort in finding positive realizations of minimum dimension.

The minimal state space realization problem for positive linear systems is that of determining a positive state space realization with the size of the system matrix $A$ as small as possible. Although the problem of finding a finite-dimensional positive state space realization has been solved, the characterization of minimality for positive systems is still an open problem. For example, when considering general linear systems, a minimal state space realization is both controllable and observable and its dimension corresponds to the rank of the Hankel matrix. A minimal positive realization, in contrast, may not be controllable and observable so that its dimension may be larger than the order of the transfer function of the system. In [5, 27] examples are provided in which the minimal dimension of a positive realization of a transfer function is much "larger" than the order of the transfer function itself. Moreover, the nonnegative rank of the Hankel matrix ${ }^{1}$ [10] is only a lower bound for the dimension of a minimal positive realization, and, as shown in [4], there are systems for which the dimension of any minimal positive realization is larger than the nonnegative rank of the Hankel matrix. A characterization of the minimality of a positive realization in terms of the nonnegative rank of the Hankel matrix has been proposed in [18] but a procedure to evaluate the minimal order of a positive realization is not available so far. Conditions for a positive realization to be minimal have been given only for special classes of positive systems such as the tree compartmental systems considered in [24] and the positive reachable systems in [13]. Results for more specific classes of positive systems are given in [17, 28]. Moreover, in [6, 9] necessary and sufficient conditions for a third order transfer functions with real poles to have a third-order (minimal) positive realization are given.

In general, only lower and upper bounds for the dimension of a minimal positive realization have been given to date. Some of these bounds can be found in $[16,17,27]$. In more detail, a lower bound for generic transfer functions and an upper bound for transfer functions with distinct real poles are provided in [16]. This latter bound is improved in [17] while in [27] a lower bound is given for a special class of transfer functions, i.e. those for which there exists a time instant at which the nonnegative impulse response is 0 and is strictly positive from that instant onwards. This bound is obtained by using diagraph techniques and extends a previous result provided in [5] by using convex cone analysis.

The aim of this letter is to provide a novel lower bound on the dimension of minimal positive realizations of impulse responses of discrete-time LTI systems by refining the

\footnotetext{
${ }^{1}$ The nonnegative rank of a nonnegative matrix is defined as the smallest inner size of a factorization of the matrix as the product of two nonnegative matrices.
} 
result provided in [16]. To this end, some properties of the dominant eigenvalues of nonnegative matrices are considered thus providing a possibly stricter bound with respect to that of Theorem 2 in [16].

\section{Preliminary results and definitions}

First note that nonnegativity of the impulse response $h(k)$ is clearly a necessary condition for the existence of a positive realization. As shown in [8], this implies that one of the dominant poles of the corresponding transfer function $H(z)=\sum_{k=1}^{\infty} h(k) z^{-k}$, that is the poles of maximum modulus, must be positive real and must have maximal multiplicity among all the dominant poles. In fact, if not, the long-term behavior of the impulse response would be oscillating thus contradicting nonnegativity assumption. Moreover, since the poles of the transfer function are a subset of the eigenvalues of the matrix $A$ of any of its positive realization, then they must be a subset of the eigenvalues of some nonnegative real matrix. In addition, as shown in Theorem 3.2 in [1], the positive real dominant pole of the transfer function must coincide with the spectral radius of the matrix $A$ of any minimal positive realization of the transfer function itself. Then, a lower bound to the dimension of a minimal positive realization of a transfer function is given by the minimum size of a nonnegative real matrix having all the poles of the transfer function among its spectrum and with spectral radius equal to the positive real dominant pole of the transfer function itself. This size is very difficult to determine since the nonnegativity constraints on the entries of the matrix $A$ impose involved limitations on the location of its eigenvalues. The problem of characterizing these limitations is known as the Nonnegative Inverse Eigenvalue Problem (NIEP) and is a long standing problem that is very difficult and, perhaps, the most prominent problem in matrix analysis [19]. In fact, a complete characterization of these limitations is known only for matrices of size $n \leq 4$ [22, 25, 30]. In this letter, a lower bound on this size is provided and to this end, the following definitions are needed.

A multiset ${ }^{2} \mathcal{M}$ will be denoted as $\mathcal{M}=(\mathcal{S}, m)$ where $\mathcal{S}$ is the set of the distinct elements in $\mathcal{M}$ and $m: \mathcal{S} \rightarrow \mathbb{Z}_{+}$is the function giving the multiplicity of each element $s \in \mathcal{S}$ in the multiset $\mathcal{M}$ as the number $m(s)$. The cardinality of a given multiset $\mathcal{M}$, which is the number of its elements (counting repetitions), is then

$$
|\mathcal{M}|=\sum_{s \in \mathcal{S}} m(s)
$$

\footnotetext{
${ }^{2} \mathrm{~A}$ multiset is a generalization of the concept of a set. It is a collection of unordered elements, where every element occurs a finite number of times. The difference between sets and multisets is in how they address multiples: a set includes any element at most once, while a multiset allows for multiple instances of the same element.
} 
Given a multiset $\mathcal{M}$ of complex numbers, its spectral radius is defined as

$$
\rho(\mathcal{M})=\max \{|s|: s \in \mathcal{S}\}
$$

Any number in the multiset $\mathcal{M}$ with modulus equal to its spectral radius $\rho(\mathcal{M})$ will be called a dominant number of the multiset and the maximal multiplicity among all the dominant numbers of $\mathcal{M}$ will be called the dominant multiplicity of the multiset and denoted as $m(\mathcal{M})$, i.e.

$$
m(\mathcal{M})=\max \{m(s): s \in \mathcal{S} \text { and }|s|=\rho(\mathcal{M})\}
$$

The set of all $k$-th roots of unity will be denoted as $\mathcal{R}_{k}$ and that of all the primitive ones $^{3}$ as $\mathcal{P}_{k}$. Every $k$-th root of unity $z$ is a primitive $h$-th root of unity for some $h \leq k$, which is the smallest positive integer $h$ such that $z^{h}=1$. In this case, $h$ is a positive divisor of $k$. Hence, the set $\mathcal{R}_{k}$ is a disjoint union of the sets $\mathcal{P}_{h}$, i.e.

$$
\mathcal{R}_{k}=\bigcup_{h \mid k} \mathcal{P}_{h}
$$

where, as usual, the notation $h \mid k$ means that $h$ goes through all the positive divisors of $k$, including 1 and $k$.

\section{Main results}

Consider a discrete-time LTI SISO system with a nonnegative impulse response $h(k)$ and assume that the reduced transfer function $H(z)=\sum_{k=1}^{\infty} h(k) z^{-k}$ has poles at location $p_{1}, \ldots, p_{n}$ with multiplicity $m_{1}, \ldots, m_{n}$, respectively. The multiset of the poles is then

$$
\mathcal{M}=(\mathcal{S}, m)
$$

with $\mathcal{S}=\left\{p_{1}, \ldots, p_{n}\right\}$ and $m\left(p_{i}\right)=m_{i}$ for $i=1, \ldots, n$. Since the coefficients of the transfer function are real numbers, then $\mathcal{M}$ is closed under complex conjugation, i.e. if $p_{i} \in \mathcal{S}$ then $\bar{p}_{i} \in \mathcal{S}$ and $m\left(\bar{p}_{i}\right)=m\left(p_{i}\right)$. Moreover, from nonnegativity of the impulse response, it follows that $\mathcal{M}$ contains its spectral radius $\rho(\mathcal{M})$ with dominant multiplicity, i.e. $\rho(\mathcal{M}) \in \mathcal{S}$ and $m(\rho(\mathcal{M}))=m(\mathcal{M})$.

A lower bound to the dimension of a minimal positive realization of a given transfer function $H(z)$ is provided by the minimum size of any nonnegative real matrix $A$ with spectral radius $\rho(A)=\rho(\mathcal{M})$ and having all the numbers of the multiset $\mathcal{M}$ among its eigenvalues. The next theorem provides a lower bound to the size of such a matrix $A$ :

\footnotetext{
${ }^{3} \mathrm{~A} k$-th root of unity $z$ is said to be primitive if it is not an $h$-th root of unity for some $h$ smaller than $k$, that is if $z^{k}=1$ and $z^{h} \neq 1$ for $h=1,2, \ldots, k-1$.
} 
Theorem 1 Let $\mathcal{M}=(\mathcal{S}, m)$ be a multiset of complex numbers that is closed under complex conjugation and contains its spectral radius with dominant multiplicity. Then $\mathcal{M}$ is a subset of the spectrum of a $N$-dimensional nonnegative real matrix $A$ with spectral radius $\rho(A)=\rho(\mathcal{M})$ if and only if the dominant numbers of the multiset are among the $r$-th roots of $\rho(\mathcal{M})^{r}$ for some positive integer $r$. Moreover, in this case, the following holds:

$$
N \geq \sum_{h \mid r} h \cdot m_{\mathcal{R}_{h}}+\sum_{\substack{p_{i} \in \mathcal{S} \\\left|p_{i}\right|<\rho(\mathcal{M})}} m\left(p_{i}\right)+\zeta
$$

where $m_{\mathcal{R}_{h}}$ is recursively defined, for $h \mid r$, as follows ${ }^{4}$

$$
m_{\mathcal{R}_{r}}=m_{\mathcal{P}_{r}}, \quad m_{\mathcal{R}_{h}}=\max \left\{0, m_{\mathcal{P}_{h}}-\sum_{\substack{h|k| r \\ k>h}} m_{\mathcal{R}_{k}}\right\}, \text { for } h<r
$$

with

$$
m_{\mathcal{P}_{h}}= \begin{cases}0 & \text { if } \nexists p_{i} \in \mathcal{S}: p_{i} / \rho(\mathcal{M}) \in \mathcal{P}_{h} \\ \max \left\{m\left(p_{i}\right): p_{i} \in \mathcal{S} \text { and } p_{i} / \rho(\mathcal{M}) \in \mathcal{P}_{h}\right\} & \text { otherwise }\end{cases}
$$

and

$$
\zeta= \begin{cases}\left\lceil-\sum_{\substack{p_{i} \in \mathcal{S} \\\left|p_{i}\right|<\rho(\mathcal{M})}} p_{i} / \rho(\mathcal{M}) \cdot m\left(p_{i}\right)-m_{\mathcal{R}_{1}}\right] & \text { if } \sum_{\substack{p_{i} \in \mathcal{S} \\\left|p_{i}\right|<\rho(\mathcal{M})}} p_{i} \cdot m\left(p_{i}\right)+\rho(\mathcal{M}) \cdot m_{\mathcal{R}_{1}}<0 \\ 0 & \text { otherwise }\end{cases}
$$

Proof. Necessity of the condition on the existence of a nonnegative real matrix $A$ immediately follows from the Perron-Frobenius Theorem [14, 29]. This theorem, which holds for irreducible nonnegative matrices, can be in fact used to determine some limitations on the location of the dominant eigenvalues of generic (reducible) nonnegative matrices. To this end, note that a reducible nonnegative matrix can be transformed, by a permutation matrix, in a nonnegative upper-triangular block matrix with irreducible or zero diagonal blocks. Since the spectrum of such a matrix is the union of the spectra of the diagonal blocks, then the dominant eigenvalues of a nonnegative real matrix $A$ of size $n$ and with spectral radius $\rho(A)$, are all the roots of $\lambda^{k}=\rho(A)^{k}$ for some (possibly more than one) values of $k \leq n$.

The sufficiency part of the proof is constructive. By assumption, all the dominant numbers in $\mathcal{M}$ are among the $r$-th roots of $\rho(\mathcal{M})^{r}$ for some positive integer $r$ and have maximal multiplicity equal to $m(\mathcal{M})$. Let $C_{r}$ denote the basic circulant matrix of dimension $r$, i.e.

$$
C_{r}=\left(\begin{array}{cc}
0 & I_{r-1} \\
1 & 0
\end{array}\right)
$$

\footnotetext{
${ }^{4}$ The notation $h|k| r$ means that $k$ goes through all the positive divisors of $r$ that are multiples of $h$.
} 
Then, the nonnegative real matrix

$$
A^{\prime}=\bigoplus_{i=1}^{m(\mathcal{M})} \rho(\mathcal{M}) \cdot C_{r}=\rho(\mathcal{M}) \cdot\left(\begin{array}{lll}
C_{r} & & \\
& \ddots & \\
& & C_{r}
\end{array}\right)
$$

has all the dominant numbers of the multiset $\mathcal{M}$ among its eigenvalues.

Moreover, any non-dominant nonnegative real number $p_{i} \in \mathcal{S}$, if any, is the eigenvalue of the nonnegative real matrix $A^{\prime}=p_{i}$ and any non-dominant negative real number $p_{j} \in \mathcal{S}$, if any, is among the eigenvalues of the nonnegative real matrix

$$
A^{\prime}=\frac{1}{2} \cdot\left(\begin{array}{ll}
\rho(\mathcal{M})+p_{j} & \rho(\mathcal{M})-p_{j} \\
\rho(\mathcal{M})-p_{j} & \rho(\mathcal{M})+p_{j}
\end{array}\right)
$$

Finally, any pair $\left(p_{k}, \bar{p}_{k}\right)$ of non-dominant conjugate complex numbers in $\mathcal{S}$, if any, is such that $p_{k} / \rho(\mathcal{M})$ lies in some Karpelevič region ${ }^{5}$. Hence, there exists a nonnegative real matrix $A^{\prime}$ with spectral radius $\rho(\mathcal{M})$ and having the pair of numbers among its eigenvalues. Then, the direct sum of all the above nonnegative realizing matrices $A^{\prime}$ is a nonnegative matrix $A$ with spectral radius $\rho(\mathcal{M})$ and having all the numbers of the multiset $\mathcal{M}$ among its eigenvalues.

A lower bound to the minimum dimension $N$ of the nonnegative real matrix $A$ can be derived taking into consideration some spectral properties of nonnegative real matrices. The first property is related to the dominant eigenvalues. In fact, as above discussed, the dominant eigenvalues of a nonnegative real matrix $A$, with spectral radius $\rho(A)$, are all the roots of $\lambda^{k}=\rho(A)^{k}$ for some, possibly more than one, values $k \in \mathbb{Z}^{+}$. Taking into account this property, it is possible to compute the minimum number of dominant eigenvalues that a nonnegative real matrix must have in order to have all the dominant numbers of the multiset $\mathcal{M}$ among its spectrum. To this end note that, since all the dominant numbers in $\mathcal{M}$ are among the $r$-th roots of $\rho(\mathcal{M})^{r}$, then each dominant number belongs to one (and only one) set

$$
\rho(\mathcal{M}) \cdot \mathcal{P}_{h}=\left\{\rho(\mathcal{M}) \cdot z: z \in \mathcal{P}_{h}\right\}
$$

\footnotetext{
${ }^{5}$ A simpler problem than NIEP was posed by Kolmogorov in 1937 [21] and it was that of determining which single complex numbers belong to the spectrum of some row stochastic matrix of dimension $n$. This problem was solved by Dmitriev and Dynkin [11] in 1946, for $n=2, \ldots, 5$, and later by Karpelevič [20] for all $n \geq 2$. They completely characterized the regions $\Theta_{n}$ of the complex plane consisting of those points which can serve as characteristic roots of $n$-dimensional row stochastic matrices. Moreover, it can be shown [26] that the spectral properties for nonnegative matrices do not differ much from those of row stochastic matrices. For example, the eigenvalues of an $n$-dimensional nonnegative matrix $A$ with positive real dominant eigenvalue $\rho$ belong to the region
}

$$
\rho \cdot \Theta_{n}=\left\{\rho z: z \in \Theta_{n}\right\}
$$


where $h$ is a positive divisor of $r$. Hence, if there are some dominant numbers $p_{i} \in$ $\rho(\mathcal{M}) \cdot \mathcal{P}_{r}$, then the matrix $A$ must necessarily have all the $r$-th roots of $\rho(\mathcal{M})^{r}$ among its eigenvalues. Moreover, the multiplicity $m_{\mathcal{R}_{r}}$ of these eigenvalues must necessarily be at least equal to the maximum multiplicity of these dominant numbers in $\mathcal{M}$, that is

$$
m_{\mathcal{R}_{r}}=\max \left\{m\left(p_{i}\right): p_{i} \in \mathcal{S} \text { and } p_{i} / \rho(\mathcal{M}) \in \mathcal{P}_{r}\right\}
$$

The same argument holds when considering dominant numbers $p_{i} \in \rho(\mathcal{M}) \cdot \mathcal{P}_{h}$ with $h$ being a positive proper divisor of $r$. However, in this case, one has to consider the fact that such dominant numbers may possibly be already among the eigenvalues of the matrix $A$ if its spectrum contains the $k$-th roots of $\rho(\mathcal{M})^{k}$ for some integer $k$ multiple of $h$. Hence, in this case, the matrix $A$ must necessarily have as eigenvalues all the $h$-th roots of $\rho(\mathcal{M})^{h}$ with multiplicity $m_{\mathcal{R}_{h}}$ given by

$$
m_{\mathcal{R}_{h}}=\max \left\{0, m_{\mathcal{P}_{h}}-\sum_{\substack{h|k| r \\ k>h}} m_{\mathcal{R}_{k}}\right\}
$$

where

$$
m_{\mathcal{P}_{h}}=\max \left\{m\left(p_{i}\right): p_{i} \in \mathcal{S} \text { and } p_{i} / \rho(\mathcal{M}) \in \mathcal{P}_{h}\right\}
$$

In conclusion, the matrix $A$ must have as eigenvalues at least all the $h$-th roots of $\rho(\mathcal{M})^{h}$ with multiplicity $m_{\mathcal{R}_{h}}$, for all the positive divisors $h$ of $r$. The total number of these eigenvalues is equal to

$$
\sum_{h \mid r} h \cdot m_{\mathcal{R}_{h}}
$$

and their sum is equal to

$$
\rho(\mathcal{M}) \cdot m_{\mathcal{R}_{1}}
$$

In fact, the sum of all the $h$-th roots of $\rho(\mathcal{M})^{h}$ is equal to zero when $h>1$.

Moreover, the matrix $A$ must have also as eigenvalues all the non dominant numbers of $\mathcal{M}$. Hence, it must have at least an additional number of eigenvalues equal to

$$
\sum_{\substack{p_{i} \in \mathcal{S} \\\left|p_{i}\right|<\rho(\mathcal{M})}} m\left(p_{i}\right)
$$

The sum of these last eigenvalues is equal to

$$
\sum_{\substack{p_{i} \in \mathcal{S} \\\left|p_{i}\right|<\rho(\mathcal{M})}} p_{i} \cdot m\left(p_{i}\right)
$$

Finally, as in Theorem 2 in [16], some properties of nonnegative matrices can be used in order to tighten the bound on the size of the matrix $A$. In particular, since the trace of a nonnegative matrix is nonnegative, then the sum of all the eigenvalues of the matrix 
$A$, which is equal to the trace of the matrix itself, has to be nonnegative. If the sum of the already considered eigenvalues, that is the sum of the values given in (1) and (2), is nonnegative, then the matrix $A$ could need no additional eigenvalues. Conversely, the matrix A must necessarily have some additional eigenvalues in order to make the sum of all its eigenvalues nonnegative. Since the maximum modulus of each eigenvalue has to be not greater than $\rho(\mathcal{M})$, then a number of additional eigenvalues at least equal to

$$
\zeta=\left\lceil-\sum_{\substack{p_{i} \in \mathcal{S} \\\left|p_{i}\right|<\rho(\mathcal{M})}} p_{i} / \rho(\mathcal{M}) \cdot m\left(p_{i}\right)-m_{\mathcal{R}_{1}}\right\rceil
$$

must be considered. This concludes the proof.

Remark 1 If the multiset $\mathcal{M}$ has a single (possibly multiple) dominant number, which is equal to $\rho(\mathcal{M})$, then the bound given in Theorem 1 reduces to that provided in Theorem 2 in [16]. In fact, if this is the case, $r=1$ and

$$
N \geq m_{\mathcal{R}_{1}}+\sum_{\substack{p_{i} \in \mathcal{S} \\\left|p_{i}\right|<\rho(\mathcal{M})}} m\left(p_{i}\right)+\zeta=m(\rho(\mathcal{M}))+\sum_{\substack{p_{i} \in \mathcal{S} \\\left|p_{i}\right|<\rho(\mathcal{M})}} m\left(p_{i}\right)+\zeta=\sum_{p_{i} \in \mathcal{S}} m\left(p_{i}\right)=|\mathcal{M}|+\zeta
$$

where $|\mathcal{M}|$ is equal to $n$ in Theorem 2 in [16]. Moreover, since

$$
\sum_{\substack{p_{i} \in \mathcal{S} \\\left|p_{i}\right|<\rho(\mathcal{M})}} p_{i} \cdot m\left(p_{i}\right)+\rho(\mathcal{M}) \cdot m_{\mathcal{R}_{1}}=\sum_{p_{i} \in \mathcal{S}} p_{i} \cdot m\left(p_{i}\right)
$$

then

$$
\zeta= \begin{cases}\left\lceil-\sum_{p_{i} \in \mathcal{S}} p_{i} / \rho(\mathcal{M}) \cdot m\left(p_{i}\right)\right] & \text { if } \sum_{p_{i} \in \mathcal{S}} p_{i} \cdot m\left(p_{i}\right)<0 \\ 0 & \text { otherwise }\end{cases}
$$

so that $\zeta$ is equal to $\xi$ in Theorem 2 in [16]. The same argument holds when the dominant numbers of the multiset $\mathcal{M}$ are all the roots of $\lambda^{k}=\rho(A)^{k}$ for some (possibly more than one) values of $k$. In fact, if this is the case,

$$
\sum_{h \mid r} h \cdot m_{\mathcal{R}_{h}}=\sum_{\substack{p_{i} \in \mathcal{S} \\\left|p_{i}\right|=\rho(\mathcal{M})}} m\left(p_{i}\right)
$$

so that the bounds given in Theorem 1 of this letter and in Theorem 2 in [16] turn out to be the same.

In general, Theorem 1 provides a stricter bound with respect to that of Theorem 2 in [16]. This is illustrated in the following example. 
Example 1 Consider the multiset $\mathcal{M}=(\mathcal{S}, m)$ with

$$
\mathcal{S}=\left\{1,-1, e^{i \frac{\pi}{3}}, e^{-i \frac{\pi}{3}}, e^{i \frac{2 \pi}{3}}, e^{-i \frac{2 \pi}{3}}, e^{i \frac{\pi}{4}}, e^{-i \frac{\pi}{4}}, e^{i \frac{3 \pi}{4}}, e^{-i \frac{3 \pi}{4}},-0.7,-0.4\right\}
$$

and

$$
m=\{2,1,1,1,2,2,2,2,1,1,1,1\}
$$

The location and multiplicity of the numbers of the multiset in the complex plane is depicted on the left of Figure 1.
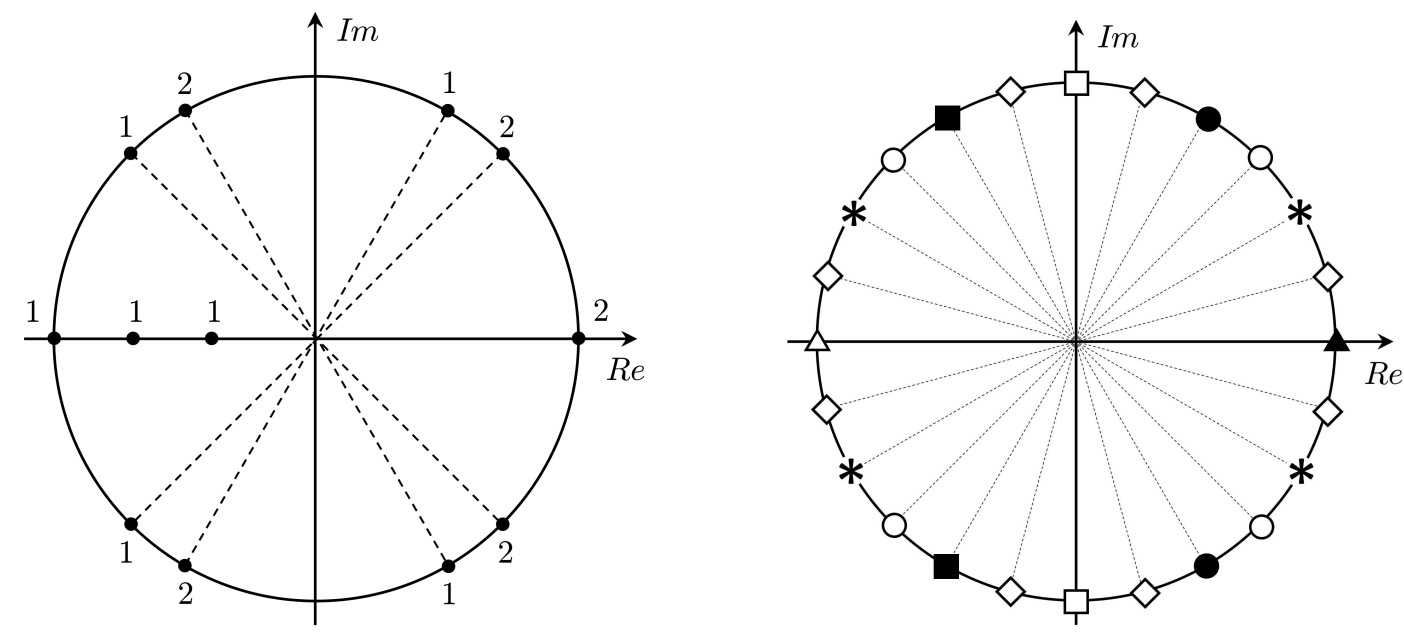

Figure 1: Location and multiplicity of the numbers of the multiset $\mathcal{M}$ with respect to the unit disk in the complex plane (left), partition of the set $\mathcal{R}_{24}$ in the sets $\mathcal{P}_{1}(\mathbf{\Lambda}), \mathcal{P}_{2}(\triangle)$, $\mathcal{P}_{3}(\boldsymbol{\bullet}), \mathcal{P}_{4}(\square), \mathcal{P}_{6}(\bullet), \mathcal{P}_{8}(\circ), \mathcal{P}_{12}(*)$ and $\mathcal{P}_{24}(\diamond)$ (right).

The cardinality of the multiset is $|\mathcal{M}|=17$ and $\rho(\mathcal{M})=1$ with $m(\mathcal{M})=2$. The sum of all the numbers of the multiset is equal to

$$
\sum_{p_{i} \in \mathcal{S}} p_{i} \cdot m\left(p_{i}\right)=0.314>0
$$

so that the lower bound provided by Theorem 2 in [16] is $N \geq 17$. Let us compute now the bound provided by Theorem 1. In this case, $r=24$ and the partition of the set $\mathcal{R}_{24}$ in the sets $\mathcal{P}_{h}$, for $h \mid 24$, is depicted on the right of Figure 1. Hence, the following hold:

$$
m_{\mathcal{P}_{24}}=m_{\mathcal{P}_{12}}=0, m_{\mathcal{P}_{8}}=2, m_{\mathcal{P}_{6}}=1, m_{\mathcal{P}_{4}}=0, m_{\mathcal{P}_{3}}=2, m_{\mathcal{P}_{2}}=1, m_{\mathcal{P}_{1}}=2 .
$$


and the values of the $m_{\mathcal{R}_{h}}$ 's can be computed as follows:

$$
\begin{aligned}
m_{\mathcal{R}_{24}} & =m_{\mathcal{P}_{24}}=0 \\
m_{\mathcal{R}_{12}} & =\max \left\{0, m_{\mathcal{P}_{12}}-m_{\mathcal{R}_{24}}\right\}=\max \{0,0\}=0 ; \\
m_{\mathcal{R}_{8}} & =\max \left\{0, m_{\mathcal{P}_{8}}-m_{\mathcal{R}_{24}}\right\}=\max \{0,2\}=2 ; \\
m_{\mathcal{R}_{6}} & =\max \left\{0, m_{\mathcal{P}_{6}}-m_{\mathcal{R}_{12}}-m_{\mathcal{R}_{24}}\right\}=\max \{0,1\}=1 ; \\
m_{\mathcal{R}_{4}} & =\max \left\{0, m_{\mathcal{P}_{4}}-m_{\mathcal{R}_{8}}-m_{\mathcal{R}_{12}}-m_{\mathcal{R}_{24}}\right\}=\max \{0,-2\}=0 ; \\
m_{\mathcal{R}_{3}} & =\max \left\{0, m_{\mathcal{P}_{3}}-m_{\mathcal{R}_{6}}-m_{\mathcal{R}_{12}}-m_{\mathcal{R}_{24}}\right\}=\max \{0,1\}=1 ; \\
m_{\mathcal{R}_{2}} & =\max \left\{0, m_{\mathcal{P}_{2}}-m_{\mathcal{R}_{4}}-m_{\mathcal{R}_{6}}-m_{\mathcal{R}_{8}}-m_{\mathcal{R}_{12}}-m_{\mathcal{R}_{24}}\right\}=\max \{0,-2\}=0 ; \\
m_{\mathcal{R}_{1}} & =\max \left\{0, m_{\mathcal{P}_{1}}-m_{\mathcal{R}_{2}}-m_{\mathcal{R}_{3}}-m_{\mathcal{R}_{4}}-m_{\mathcal{R}_{6}}-m_{\mathcal{R}_{8}}-m_{\mathcal{R}_{12}}-m_{\mathcal{R}_{24}}\right\}=0 .
\end{aligned}
$$

Consequently, a nonnegative real matrix with spectral radius $\rho(\mathcal{M})=1$ and having all the dominant numbers of $\mathcal{M}$ as eigenvalues must have a dimension at least equal to

$$
\sum_{h \mid 24} h \cdot m_{\mathcal{R}_{h}}=25
$$

Moreover, the nonnegative real matrix A must have also the two non-dominant numbers of $\mathcal{M}$, i.e. -0.7 and -0.4 , as eigenvalues. In fact,

$$
\sum_{\substack{p_{i} \in \mathcal{S} \\\left|p_{i}\right|<1}} m\left(p_{i}\right)=2
$$

Finally, in this case,

$$
\sum_{\substack{p_{i} \in \mathcal{S} \\\left|p_{i}\right|<1}} p_{i} \cdot m\left(p_{i}\right)+\rho(\mathcal{M}) \cdot m_{\mathcal{R}_{1}}=-1.1
$$

so that $\zeta=2$. In conclusion, a nonnegative real matrix $A$ with $\rho(A)=\rho(\mathcal{M})=1$ and having all the 17 numbers of $\mathcal{M}$ as eigenvalues must have a dimension $N$ at least equal to 29.

Note that the upper bounds on the minimal dimension of a positive realization provided in [16] and [17] are valid for transfer functions with distinct real poles. For such transfer functions, as discussed in Remark 1, the lower bound given in Theorem 1 coincides with that of Theorem 2 in [16] and hence the result here provided does not reduce the gap between these lower and upper bounds.

Moreover, as reported in the introduction, the lower bound given in [27] is developed for a very special class of transfer functions. The bound is expected to be less conservative than the one proposed in this letter since it takes into account nonnegativity constraints on the entries not only of the dynamic matrix $A$ but of the vectors $b$ and $c$ also. This seems to be true for transfer functions with distinct real poles, as for the examples provided in [27]. Nevertheless, as the following example makes clear, this is not always the case when transfer functions with dominant complex poles are considered. 
Example 2 Consider the fifth order transfer function

$$
H(z)=\frac{1}{z-0.9}+\frac{0.045 \sqrt{2}}{z^{2}-0.9 \sqrt{2} z+0.81}-\frac{r}{z-0.8}+\frac{r}{z-0.7}
$$

whose impulse response is

$$
h(k)=0.9^{k-1}+0.1 \cdot 0.9^{k-1} \sin [(k-1) \pi / 4]-r\left(0.8^{k-1}-0.7^{k-1}\right), \quad k>0
$$

If $r=9^{7} /\left(10 \cdot\left(8^{6}-7^{6}\right)\right) \approx 3.31$, then $h(k)$ is nonnegative, $h(7)=0$ and $h(k)>0$ for all $k>7$. The impulse response $h(k)$ is depicted in Figure 2. Hence the transfer function

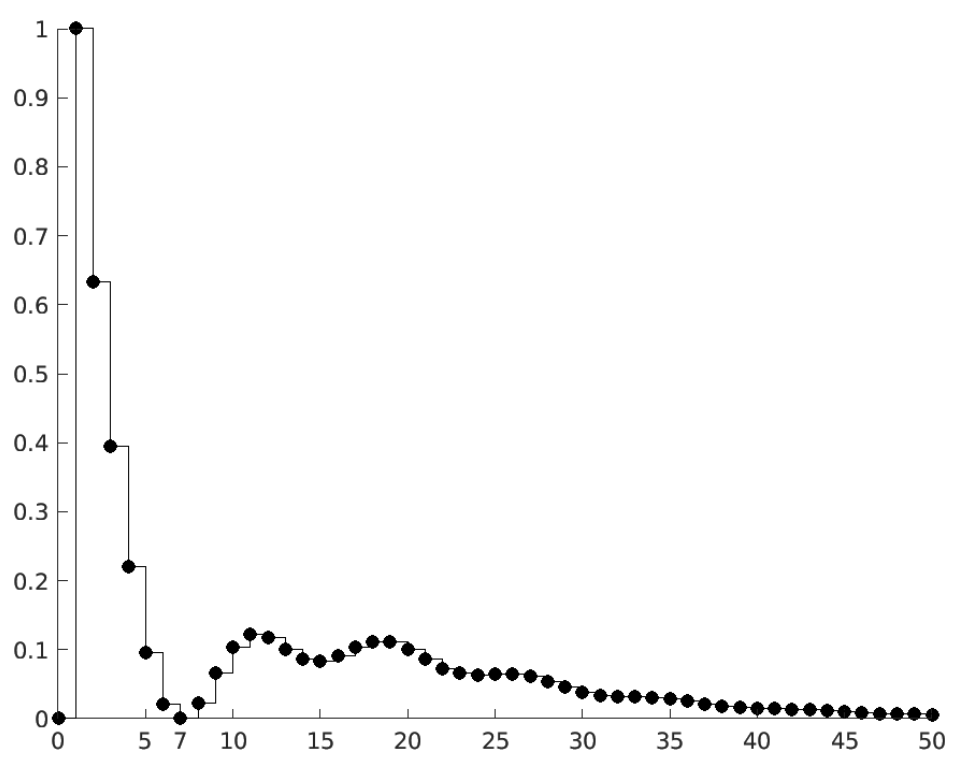

Figure 2: The impulse response $h(k)$ considered in Example 2.

belongs to the class considered in [27]. In this case, however, the lower bound given there results in a value $N=4$ (i.e. the minimum value of $N$ such that $N^{2}-2 N+2 \geq 7$ ) that is actually lesser than the order of the transfer function itself.

Let us compute the bound provided by Theorem 1. The multiset of the poles of the transfer function is $\mathcal{M}=(\mathcal{S}, m)$ with

$$
\mathcal{S}=\left\{0.9,0.9 \cdot e^{i \frac{\pi}{4}}, 0.9 \cdot e^{-i \frac{\pi}{4}}, 0.8,0.7\right\}
$$

and $m\left(p_{i}\right)=1$ for all $i$. In this case, $\rho(\mathcal{M})=0.9, r=8$ and $m_{\mathcal{P}_{8}}=1, m_{\mathcal{P}_{4}}=m_{\mathcal{P}_{2}}=0$, $m_{\mathcal{P}_{1}}=1$. Consequently, the values of the $m_{\mathcal{R}_{h}}$ 's can be computed, for $h \mid 8$, as:

$$
\begin{aligned}
& m_{\mathcal{R}_{8}}=m_{\mathcal{P}_{8}}=1 \\
& m_{\mathcal{R}_{4}}=\max \left\{0, m_{\mathcal{P}_{4}}-m_{\mathcal{R}_{8}}\right\}=\max \{0,-1\}=0 ; \\
& m_{\mathcal{R}_{2}}=\max \left\{0, m_{\mathcal{P}_{2}}-m_{\mathcal{R}_{4}}-m_{\mathcal{R}_{8}}\right\}=\max \{0,-1\}=0 ; \\
& m_{\mathcal{R}_{1}}=\max \left\{0, m_{\mathcal{P}_{1}}-m_{\mathcal{R}_{2}}-m_{\mathcal{R}_{4}}-m_{\mathcal{R}_{8}}\right\}=\max \{0,0\}=0 .
\end{aligned}
$$


Moreover, the nonnegative real matrix A must have also the two non-dominant numbers of $\mathcal{M}$, i.e. 0.8 and 0.7 , as eigenvalues. Finally, in this case, $\zeta=0$ so that a nonnegative real matrix $A$ with $\rho(A)=\rho(\mathcal{M})=0.9$ and having all the 5 numbers of $\mathcal{M}$ as eigenvalues must have a size $N$ not less than 10 .

As a final consideration, an interesting question to investigate is whether and how this result can be extended to multi-input multi-output (MIMO) systems. To this aim, it is first necessary to note that the proof of Theorem 3.2 in [1] can be easily extended to the case of MIMO systems. Hence, the spectral radius of the matrix $A$ of a minimal positive realization of a given transfer function matrix $H(z)$ must coincide with the positive real dominant pole of the transfer function matrix itself. Then, Theorem 1 can be used to provide a lower bound to a minimal positive realization taking into consideration that, in the MIMO case, the multiplicity of a pole is defined as its multiplicity as a root in the characteristic polynomial of a minimal realization of $H(z)$, which is the least common multiple of the denominators of all possible minors in $H(z)$.

\section{Conclusion}

In this letter, a lower bound on the dimension of minimal positive realizations of transfer functions of discrete-time LTI systems is provided. This bound is a refinement of a result provided in [16] and, in general, it is less conservative. The bound depends on the location of the poles of the transfer function and exploits the spectral properties of nonnegative matrices. It would be interesting to extend the upper bound presented in [16] and [17] to the case of more general transfer function but this task seems to be difficult since also nonnegativity of vectors $c$ and $b$ must be considered.

\section{References}

[1] B. D. O. Anderson, M. Deistler, L. Farina, and L. Benvenuti, Nonnegative realization of a linear system with nonnegative impulse response. IEEE Transactions on Circuits and Systems I: Fundamental Theory and Applications, 43(2):134-142, 1996.

[2] B. D. O. Anderson, The realization problem for hidden Markov models. Mathematics of Control, Signals, and Systems, 12:80-120, 1999.

[3] L. Benvenuti and L. Farina, Discrete-time filtering via charge routing networks. Signal Processing, 49(3):207-215, 1996.

[4] L. Benvenuti and L. Farina, A note on minimality of positive realizations. IEEE Transactions on Circuits and Systems I: Fundamental Theory and Applications, 45(6):676-677, 1998. 
[5] L. Benvenuti and L. Farina, An example of how positivity may force realizations of 'large' dimension. System \&6 Control Letters, 36(4):261-266, 1999.

[6] L. Benvenuti, L. Farina, B. D. O. Anderson, and F. De Bruyne, Minimal positive realizations of transfer functions with positive real poles. IEEE Transactions on Circuits and Systems I: Fundamental Theory and Applications, 47(9):1370-1377, 2000.

[7] L. Benvenuti and L. Farina, The design of fiber-optic filters. IEEE/OSA Journal of Lightwave Technology, 19(9):1366-1375, 2001.

[8] L. Benvenuti and L. Farina, A tutorial on the positive realization problem. IEEE Transactions on Automatic Control, 49:651-664, 2004.

[9] L. Benvenuti, Minimal positive realizations of transfer functions with real poles, IEEE Transactions on Automatic Control, 58:1013-1017, 2013.

[10] J. E. Cohen and U. G. Rothblum, Nonnegative ranks, decompositions, and factorizations of nonnegative matrices, Linear Algebra and its Applications, 190:149-168, 1993.

[11] N. Dmitriev, E. Dynkin, On characteristic roots of stochastic matrices, Izvestiya Akademii Nauk SSSR Seriya Matematicheskaya, 10:167-184, 1946.

[12] L. Farina, On the existence of a positive realization, System $\&$ Control Letters, 28:219-226, 1996.

[13] L. Farina, Minimal order realizations for a class of positive linear systems, Journal of the Franklin Institute, 333B:893-900, 1996.

[14] G. Frobenius, Über matrizen aus nicht negativen elementen, Sitzungsberichte der Königlich Preussischen Akademie der Wissenschaften. 456-477, 1912.

[15] A. Gersho and B. Gopinath, Charge-routing networks. IEEE Transactions on Circuits and Systems, 26(1):81-92, 1979.

[16] C. Hadjicostis, Bounds on the size of minimal nonnegative realization for discretetime lti systems. System \& Control Letters, 37(1):39-43, 1999.

[17] A. Halmschlager and M. Matolcsi, Minimal positive realizations for a class of transfer functions. IEEE Transactions on Circuits and Systems II: Express Briefs, 52(4):177180, 2005.

[18] J. M. van den Hof, Realization of positive linear systems. Linear Algebra and its Applications, 256:287-308, 1997. 
[19] C. R. Johnson, C. Marijuán, P. Paparella, M. Pisonero, The NIEP, Operator Theory: Advances and Applications, 267:199-220, 2018.

[20] F. I. Karpelevič, On the characteristic roots of matrices with nonnegative elements, Izvestiya Akademii Nauk SSSR Seriya Matematicheskaya, 15:361-383, 1951.

[21] A. N. Kolmogorov, Markov chains with a countable number of possible states, Byull. Moskov Gosudarstvennogo Universiteta. Matematika i Mehanika, 1(3):1-16, 1937.

[22] R. Loewy and D. London, A note on an inverse problem for nonnegative matrices, Linear and Multilinear Algebra, 6: 83-90, 1978.

[23] D. G. Luenberger, Positive linear systems. In Introduction to dynamic systems: theory, models, and applications. John Wiley \& Sons, New York, 1979.

[24] H. Maeda, S. Kodama, and F. Kajiya, Compartmental system analysis: realization of a class of linear systems with physical constraints. IEEE Transactions on Circuits and Systems, 24(1):8-14, 1977.

[25] M. E. Meehan, Some results on matrix spectra, PhD thesis, National University of Ireland, Dublin, 1998.

[26] H. Minc, Nonnegative Matrices, John Wiley \& Sons, New York, 1988.

[27] B. Nagy and M. Matolcsi, A lowerbound on the dimension of positive realizations. IEEE Transactions on Circuits and Systems I: Fundamental Theory and Applications, 50(6):782-784, 2003.

[28] B. Nagy and M. Matolcsi, Minimal positive realizations of transfer functions with nonnegative multiple poles. IEEE Transactions on Automatic Control, 50(9):14471450, 2005.

[29] O. Perron, Zur theorie der matrizen, Mathematische Annalen, 64:248-263, 1907.

[30] J. Torre-Mayo, M. R. Abril-Raymundo, E. Alarcia-Estévez, C. Marijuán, M. Pisonero, The nonnegative inverse eigenvalue problem from the coefficients of the characteristic polynomial. EBL digraphs, Linear Algebra and its Applications, 426:729-773, 2007.

[31] M. Vidyasagar, The complete realization problem for hidden Markov models: a survey and some new results. Mathematics of Control, Signals, and Systems, 23:165, 2011. 